\title{
X-linked Retinoschisis Discovered after Congenital Cataract Surgery
}

\author{
Ayoub Redouan, Ismail Essemlali, Loubna El Maaloum, Bouchra Allali, Asmae El Kettani
}

\section{ABSTRACT}

$\mathrm{X}$-linked retinoschisis (XLRS) is a rare, hereditary, and bilateral vitreoretinal degeneration, associating maculopathy and vitreoretinopathy. We report the case of $\mathrm{X}$-linked retinoschisis diagnosed after congenital cataract surgery in a child.

Published Online: November 30, 2020

ISSN: $2593-8339$

DOI: $10.24018 /$ ejmed.2020.2.6.584

Keywords: Retinoschisis, Optical Coherence Tomography, vitreoretinal degeneration.

\section{Ayoub Redouan*}

Hassan II University, Morocco. (e-mail: drayoubredouan@ ${ }^{\circledR}$ gmail.com) Ismail Essemlali

Hassan II University, Morocco. Loubna EI Maaloum

Hassan II University, Morocco. Bouchra Allali

Hassan II University, Morocco. Asmae El Kettani

Hassan II University, Morocco.

*Corresponding Author

\section{INTRODUCTION}

$\mathrm{X}$-linked retinoschisis is a rare, hereditary, and bilateral vitreoretinal degeneration, associating maculopathy and vitreoretinopathy.

\section{CASE REPORT}

We report the case of $\mathrm{X}$-linked retinoschisis diagnosed in the aftermath of a child operated on for congenital unilateral OD cataract.

Child aged 5 years, followed for unilateral congenital cataract of the operated OD, and implanted in the sac with post rhexis and ant vitrectomy. The evolution was marked by a deep amblyopia of the average OD of the OG despite an OC and a TTT of well-conducted amblyopia.

The anterior segment was normal on the left and on the right: the IOL was in place, no secondary cataract and the intra ocular pressure was normal.

In the ocular fundus:

Right eye: there is an inter-papillo-macular tractional membrane with tortuosity and stretching of the vessels. (Fig. 1).

Left eye: Wheel raillon aspect (Fig. 2).

OCT: more marked and more extensive macular retinoschisis on the right (Fig. 3).

ERG: wave « $\mathrm{b}$ » is negative and wave «a» is positive. (Fig. 4).

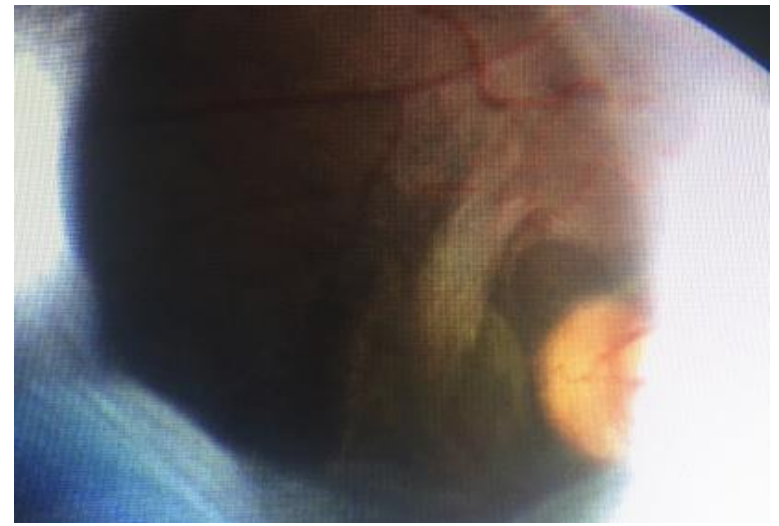

Fig. 1. Right eye: An inter-papillo-macular tractional membrane with tortuosity and stretching of the vessels.

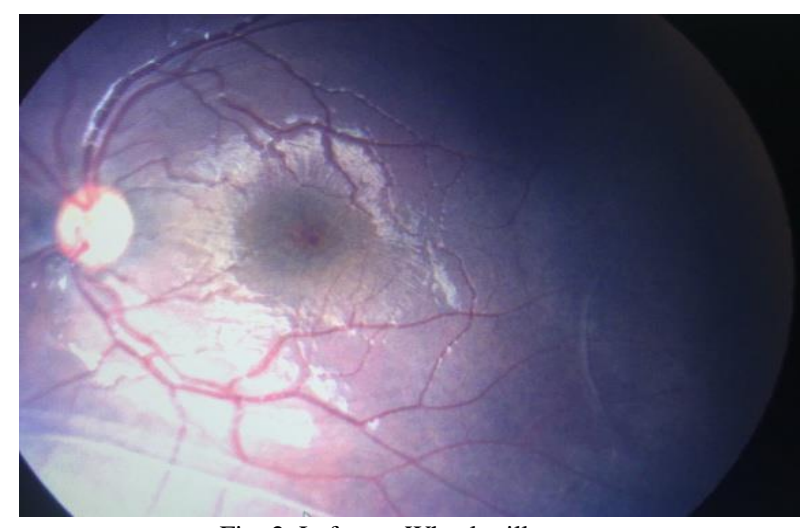

Fig. 2. Left eye: Wheel raillon aspect 


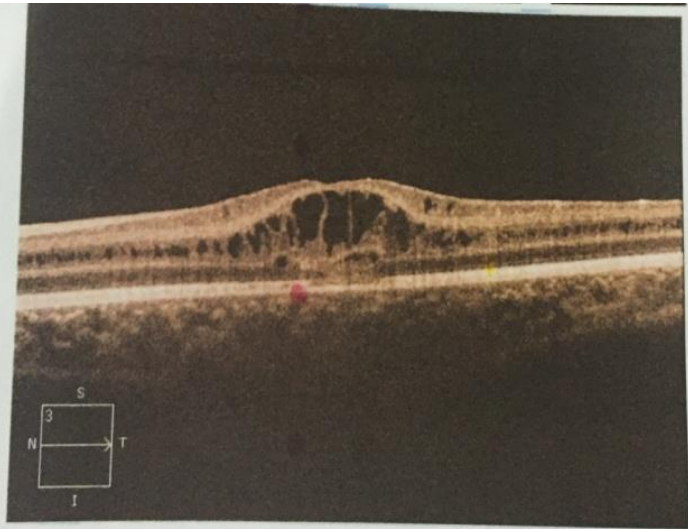

Fig. 3. More marked and more extensive macular retinoschisis on the right eye.

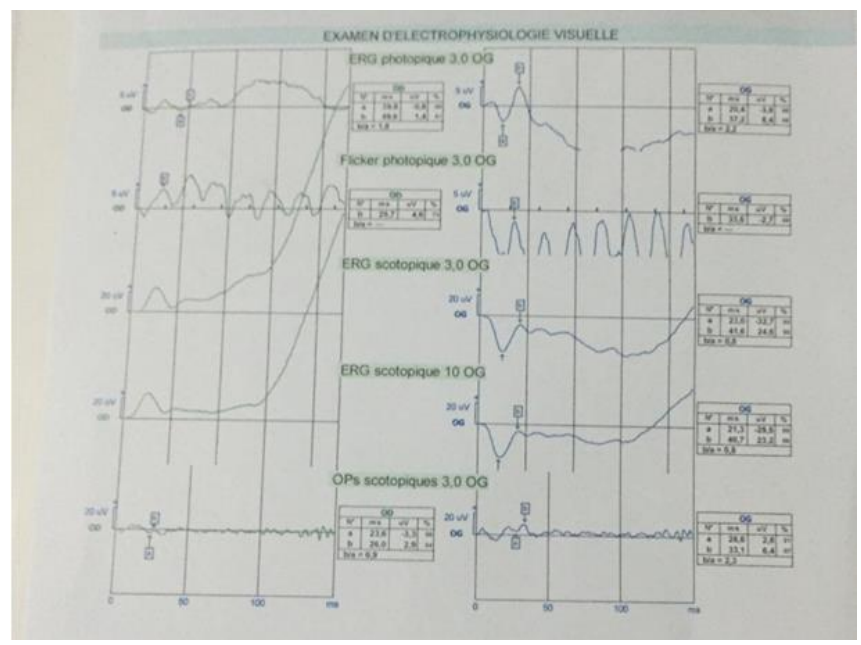

Fig. 4. A negative wave $« \mathrm{~b} »$ and a positive wave $« \mathrm{a} »$.

\section{DISCUSSION}

The most common mode of discovery of XLRS is decrease in Visual Acuity [1], it often affects males with rare cases in females. [2,3].

It is characterized by still present bilateral foveal retinoschisis, peripheral retinoschisis, perivascular cuffs, vitreous veils, and pigmento-atrophic changes [4]. Other anomalies can be associated: axile hyperopia, strabismus, and cataract [5].

The electroretinogram is still disturbed and shows a pattern characteristic of the condition [6].

The appearance on OCT of XLRS is very evocative with a superficial cleavage in the form of hyporeflective cavities which are particularly predominant in the macula [7].

The evolution is marked by a stable VA during the first 2 decades [8].

\section{CONCLUSION}

$\mathrm{X}$-linked retinoschisis is a rare pathology, its clinical presentation is variable, and the diagnosis is difficult in children, but can be precipitated by the occurrence of complications. The forms diagnosed in children are more often severe and the prognosis remains poor regardless of the age of diagnosis, hence the interest of genetic counseling

\section{REFERENCES}

[1] Samantha R. De Silva, Progress in Retinal and Eye Research, https://doi.org/10.1016/j.preteyeres.2020.100898.

[2] Önen M, Zor K, Küçük E, Yıldırım G. X-Linked Retinoschisis in Females in a Consanguineous Family: A Rare Entity. Turk J Ophthalmol, 2020; 50:252-254.

[3] A. Altun et al, A Female Case of X-Linked Retinoschisis with Macular Hole Bilaterally, Case Reports in Ophthalmological Medicine / 2020 Article ID 8824995 https://doi.org/10.1155/2020/8824995.

[4] Grigg, J.R., Hooper, C.Y., Fraser, C.L. et al. Outcome measures in juvenile X-linked retinoschisis: A systematic review. Eye, 34, 17601769 (2020). https://doi.org/10.1038/s41433-020-0848-6.

[5] Chunjie Chen, Yue Xie, Tengyang Sun, Lu Tian, Ke Xu, Xiaohui Zhang, and Yang Li Clinical findings and RS1 genotype in 90 Chinese families with X-linked retinoschisis. Mol Vis. 2020; 26: 291 298.

[6] J. Jason McAnany, Jason C. Park, Gerald A. Fishman, and Frederick T. Collison Full-Field Electroretinography, Pupillometry, and Luminance Thresholds in X-Linked Retinoschisis. Investigative Ophthalmology \& Visual Science, June 2020, Vol. 61, 53. doi: https://doi.org/10.1167/iovs.61.6.53.

[7] Chong, Y.J., Price-Hunt, G. \& Denniston, A. Adult Presentation of XLinked Retinoschisis: Patient and Physician Perspectives. Ophthalmo Ther, 9, 15-19 (2020). https://doi.org/10.1007/s40123-020-00233-1.

[8] Besada, E., \& Frauens, B. (2019). Long-term stability of an asymptomatic optic pit with foveolar retinoschisis. Clinical and Experimental Optometry. doi:10.1111/cxo.12920. 